\title{
A Two-Stage Filter for High Density Salt and Pepper Denoising
}

\author{
Dang N. H. Thanh, Nguyen Hoang Hai, Le Minh Hieu, V. B. Surya Prasath, João Manuel R. S. Tavares
}

\begin{abstract}
Image restoration is an important and interesting problem in the field of image processing because it improves the quality of input images, which facilitates postprocessing tasks. The salt-and-pepper noise has a simpler structure than other noises, such as Gaussian and Poisson noises, but is a very common type of noise caused by many electronic devices. In this article, we propose a two-stage filter to remove high-density salt-and-pepper noise on images. The range of application of the proposed denoising method goes from low-density to high-density corrupted images. In the experiments, we assessed the image quality after denoising using the peak signal-to-noise ratio and structural similarity metric. We also compared our method against other similar state-of-the-art denoising methods to prove its effectiveness for salt and pepper noise removal. From the findings, one can conclude that the proposed method can successfully remove super-high-density noise with noise level above $90 \%$.
\end{abstract}

Index Terms-Denoising, Salt and Pepper noise, image restoration, image processing, image quality assessment.

\section{INTRODUCTION}

In image processing, denoising is one of the most important preprocessing tasks to improve image quality. There are several types of noise, such as Gaussian noise, Poisson noise, Speckle noise, and impulse noise. The salt and pepper ( $\mathrm{SnP})$ noise is a simple type of impulse noise $[1,2]$, where the noisy pixel has only two possible values: 255 - white pixel or salt pixel, and 0

Manuscript received July 30, 2019; revised March 21, 2020; accepted

Dang N. H. Thanh, Department of Information Technology, School of Business Information Technology, University of Economics Ho Chi Minh city, Vietnam. Email: thanhdnh@ueh.edu.vn.

Nguyen Hoang Hai, Vietnam-Korea University of Information and Communication Technology - The University of Danang, Vietnam. Email: nhhai@vku.udn.vn.

Le Minh Hieu, Department of Economics, University of Economics The University of Danang, Vietnam. Email: hieulm@due.edu.vn.

V. B. Surya Prasath, Division of Biomedical Informatics, Cincinnati Children's Hospital Medical Center, Cincinnati, OH USA; Department of Pediatrics, University of Cincinnati, OH USA; Department of Biomedical Informatics, College of Medicine, University of Cincinnati, OH USA; Department of Electrical Engineering and Computer Science, University of Cincinnati, OH USA. Email: prasatsa@uc.edu.

João Manuel R. S. Tavares, Instituto de Ciência e Inovação em Engenharia Mecânica e Engenharia Industrial, Departamento de Engenharia Mecânica, Faculdade de Engenharia, Universidade do Porto, Porto, Portugal. Email:tavares@fe.up.pt. (zero) - black pixel or pepper pixel, for an 8-bit grayscale image. We must notice that, for a natural image containing SnP noise, the gray values of noisy pixels are always different from the boundary values of the range of gray values.

There are several proposed approaches to remove $\mathrm{SnP}$ noise, such as based on regularization, filtering, principal component analysis, and machine learning. Regularization by total variation (TV) based on $\mathrm{L}^{1}$ norm is an effective approach to remove this type of noise $[1,3,4]$. Thanh et al. proposed an adaptive TV-L ${ }^{1}$ (ATV1) denoising method $[2,5]$ with adaptive regularization parameter estimation that attains better denoising results than traditional TV-L ${ }^{1}$ and works well on high-density noise (up to $80 \%$ ) corrupted images. As to denoising filters, nonlinear filters $[6,7,8,9]$ are effective methods to remove $\mathrm{SnP}$ noise, and, because they are non-iterative approaches, their implementing speed does not depend on a tolerance parameter. Nonlinear filters are usually developed based on median-based filters, mean-based filters or Wiener-based filters. In recent years, some nonlinear filters were proposed to remove $\mathrm{SnP}$ noise, such as the adaptive median filter (AMF) [10], the noise adaptive fuzzy switching median filter (NAFSMF) [11], decision based unsymmetric trimmed median filter (DBUTMF) $[12,13]$, the modified DBUTMF (MDBUTMF) [14], the basedon-pixel density filter (BPDF) [15], the different applied median filter (DAMF) [16], the adaptive frequency median filter (AFMF) [17] and the iterative mean filter (IMF) [18]. $\mathrm{AMF}$ and BPDF remove noise at low density very well. However, they fail for high-density noise (higher than 50\%): AMF makes the image too blurred and BPDF destroys the input image structure. NAFSMF and MDBUTMF are effective filters for high-density noise. NAFSMF is a recursive filter with two stages: noise detection and filtering, and it removes noise based on a fuzzy function. Both DBUTMF and MDBUTMF work well on high-density noise; however, if the considered window only has salt pixel and/or pepper pixel, the trimmed median of DBUTMF cannot be evaluated. MDBUTMF overcomes this drawback and is more effective than DBUTMF. On the other hand, DBA (Decision Based Algorithm) [19], ACWMF (Adaptive Centre Weighted Median Filter) [20], NASNLM (Noise Adaptive Switching Nonlocal Mean) [21] and FSD (Type-2 Fuzzy SnP Denoising) [22] are also effective state-ofthe-art filters for SnP noise including high-density noise. 
In this article, we propose a new filter, which is designated as a two-stage filter (TSF), to remove high-density SnP noise in images. TSF includes two main stages: high-density noise reduction and low-density noise removal. In the experiments, we used the UC-Berkeley dataset (BSDS dataset), and we compared the denoising results obtained by the proposed method against the ones obtained by ATV1, DBA, ACWMF, MDBUTMF, NAFSM, NASNLM, BPDF, FSD, DAMF, and AFMF methods. The denoising quality was assessed based on the peak signal-to-noise ratio and similarity metric.

The rest of the article is organized as follows. Section II describes the proposed salt and pepper denoising method. Section III presents the experimental results and their discussion. Section IV concludes the article.

\section{Proposed Salt and Pepper Denoising Method}

\section{A. Definitions and Notions}

Let $\left[u_{i j}\right]_{m \times n^{\prime}}\left[v_{i j}\right]_{m \times n}$ be an original image and a corrupted image by SnP noise, respectively, where $m$ and $n$ are the number of pixels by the height and by the width of an image.

Definition 1. Denote the dynamic range of the gray values of pixels (pixels value) by $\left[\delta_{\min }, \delta_{\max }\right]$, i.e. $\delta_{\min } \leq u_{i j} \leq$ $\delta_{\max }$. Therefore, the corrupted image is given by:

$$
v_{i j}=\left\{\begin{array}{ll}
\delta_{\min } & \text { with probability } p \\
\delta_{\max } & \text { with probability } q \\
u_{i j} & \text { with probability } 1-(p+q)
\end{array},\right.
$$

where $0 \leq p, q \leq 1$. Let denoting an SnP noise level (or an SnP noise ratio) as $r=p+q$, and considerer that if $r$ is even, then $p=q$.

Definition 2. Let $d \geq 1$ be an integer number. A window with size $(2 d+1)$ centred at a pixel $(i, j)$ is defined as:

$$
W_{d}^{(i, j)}=\{(k, l):|k-i| \leq d,|l-j| \leq d\} .
$$

Definition 3. We denote a set of pixels of an image domain $\Omega$ of a corrupted image $v$ by $\mathcal{N}(\Omega)$. Let consider a pixel $(i, j)$ of the image domain: $(i, j) \in \mathcal{N}(\Omega)$, then:

a) Pixel $(i, j)$ is considered a strictly noisy pixel (by the salt and pepper noise) if $v_{i j} \in\left\{\delta_{\min }, \delta_{\max }\right\}$. We denote the set of strictly noisy pixels of the image $v$ by $\widehat{\mathcal{N}}(\Omega)$.

b) Pixel $(i, j)$ is considered an approximately noisy pixel if its gray value $v_{i j} \in\left(\delta_{\min }, \delta_{\min }+\delta\right) \cup\left(\delta_{\max }-\delta, \delta_{\max }\right)$, where $\delta>0$. We denote the set of approximately noisy pixels of the image $v$ by $\tilde{\mathcal{N}}(\Omega)$.

c) If a pixel is not a noisy pixel (strictly and approximately), then it is considered an original pixel and be denoted by $\ddot{\mathcal{N}}(\Omega)$.

d) If a pixel is not a strictly noisy pixel, then it is considered a weakly original pixel and be denoted by $\widetilde{\mathcal{N}}(\Omega)$.

Proposition 1. For every corrupted image by SnP noise $v$, we always have the following equalities:

$$
\begin{gathered}
\mathcal{N}(\Omega)=\widehat{\mathcal{N}}(\Omega) \cup \widetilde{\mathcal{N}}(\Omega) \cup \ddot{\mathcal{N}}(\Omega), \\
\mathcal{N}(\Omega)=\widehat{\mathcal{N}}(\Omega) \cup \overline{\mathcal{N}}(\Omega) .
\end{gathered}
$$

Definition 4. Let $(i, j)$ be original pixels. Let $\ell_{k} \in$ $\left[\delta_{\text {min }}, \delta_{\text {max }}\right]$ be the values of the gray levels with $k=1,2,3, \ldots$ Let $\mathcal{L}_{k}=\left\{(i, j), v_{i j}=\ell_{k}\right\}$, then:

a) If the cardinality $\operatorname{card}\left(\mathcal{L}_{k}\right)>1$, then pixels $(i, j)$ are considered as repetitive pixels.

b) Set $\mathcal{L}_{\epsilon}=\left\{(i, j): \operatorname{card}\left(\mathcal{L}_{\epsilon}\right)=\max _{k}\left\{\operatorname{card}\left(\mathcal{L}_{k}\right)\right\}\right\}$, then pixels $(i, j)$ are designated as maximum repetitive pixels. The corresponding pixel values $v_{\mathcal{L}_{\epsilon}}=\left\{v_{i j}:(i, j) \in \mathcal{L}_{\epsilon}\right\}$ are considered as maximum repetitive pixel values.

\section{B. Proposed Denoising Method}

The proposed method includes two stages: I) the first stage reduces the high-density noise based on the median of the weakly original pixels, and II) the second one that removes the low-density noise based on the median of the maximum repetitive pixel values. The maximum repetitive pixel values are more effective to remove low-density noise than the median of weakly original pixels. However, for high-density noise, the median of weakly original pixels does not reflect the value of corrupted pixels correctly because there are very few original pixels. Therefore, a transformation from high-density noise to low-density noise is necessary to apply the median of maximum repetitive pixel values.

Algorithm 1. Two-Stage Filter for Removing High-Density Salt and Pepper Noise.

Input: Noisy image $v$.

Output: Restored image $u$.

Function $u=\operatorname{TSF}(v)$

Initialize $d_{\max }, \phi \leftarrow v$.

For $(i, j) \in \widehat{\mathcal{N}}(\phi)$

$$
\begin{aligned}
& \text { If }\left(\exists(s, t) \in \overline{\mathcal{N}}\left(W_{1}^{(i, j)}\right)\right) \text { Then } \\
& \quad M \leftarrow \text { Eliminate all pixels }(k, l) \in \widehat{\mathcal{N}}\left(W_{1}^{(i, j)}\right) . \\
& \quad \phi(i, j) \leftarrow \text { median }\left\{\phi_{M}\right\} . \\
& \text { Else If }\left(\exists(s, t) \in \overline{\mathcal{N}}\left(W_{3}^{(i, j)}\right)\right) \text { Then } \\
& \quad M \leftarrow \text { Eliminate all pixels }(k, l) \in \widehat{\mathcal{N}}\left(W_{3}^{(i, j)}\right) . \\
& \quad \phi(i, j) \leftarrow \text { median }\left\{\phi_{M}\right\} . \\
& \text { End. }
\end{aligned}
$$

End.

$$
\begin{aligned}
& \text { Set } u \leftarrow \phi . \\
& \text { For }(i, j) \in \widehat{\mathcal{N}}(u) \\
& d \leftarrow 1 . \\
& \quad \text { Repeat } \\
& \quad \text { If }\left(\exists(s, t) \in \widetilde{\mathcal{N}}\left(W_{d}^{(i, j)}\right) \cup \widetilde{\mathcal{N}}\left(W_{d}^{(i, j)}\right)\right) \text { Then } \\
& \quad u(i, j) \leftarrow \operatorname{median}\left\{\phi_{\mathcal{L}_{\epsilon}}\right\} . \\
& \quad \text { Else } \\
& \quad d \leftarrow d+1 . \\
& \text { End. } \\
& \text { Until }\left(d>d_{\text {max }}\right) . \\
& \text { End. }
\end{aligned}
$$

In the proposed method, for the first stage of reducing the high-density noise, two windows with fixed sizes are used: $3 \times 3$ or $7 \times 7$. Hence, the proposed method still presents the advantages of the high-density denoising methods. 
After the reducing noise step of the first stage, it is implemented in the second stage to remove the remaining noise by the median of the maximum repetitive pixel values. In this stage, we use dynamic windows with flexible sizes. The size of these dynamic windows is enlarged gradually until there is at least a weak original pixel in the considered window. The proposed method in the second stage uses windows with sizes $3 \times 3,5 \times 5,7 \times 7$, etc. The limit size can be enlarged to the minimum value of the image width and the image height, i.e. $d_{\max }=\lfloor\min \{m, n\} / 2\rfloor$, where $[\cdot]$ is floor operator.

Details of the proposed denoising method are given in Algorithm 1; note that, $\phi_{M}=\left\{\phi_{i j}:(i, j) \in M\right\}$.

Theorem 1. The complexity of Algorithm 1 is $\mathcal{O}\left(n^{3}\right)$.

Proof. Let $a=|\widehat{\mathcal{N}}(\phi)|, b=|\widehat{\mathcal{N}}(u)|$, where $|\cdot|$ is cardinality (number of elements of a set). Maximum number of operations (MNO) of the first stage and MNO of the second stage are:

$$
\begin{gathered}
f_{1}=\max \{(3 \times 3-1) a,(7 \times 7-1) a\}=48 a, \\
f_{2}=b\left[\frac{\min (m, n)}{2}\right] .
\end{gathered}
$$

Hence, MNO of Algorithm 1 is:

$$
f=f_{1}+f_{2}=48 a+b\left\lfloor\frac{\min (m, n)}{2}\right\rfloor .
$$

Let consider that $m=n$. In the worst case, $a=b=n^{2}$ (i.e. all pixels are corrupted). Then, we have:

$$
f=48 n^{2}+n^{2}\left\lfloor\frac{n}{2}\right\rfloor \text {. }
$$

Therefore, the complexity of Algorithm 1 is:

$$
\mathcal{O}(f)=\mathcal{O}\left(48 n^{2}+\frac{n^{3}}{2}\right)=\mathcal{O}\left(n^{3}\right) .
$$

\section{EXPERIMENTAL RESULTS AND DISCUSSION}

We conducted the experiments of the proposed method for $\mathrm{SnP}$ denoising on MATLAB. The configuration of the used computing system was Intel Core i5, 1.6 GHz, 4 GB $2295 \mathrm{MHz}$ DDR3 RAM and Windows 10 Pro.

\section{A. Image Quality Assessment Metrics}

To evaluate the image quality after denoising, we used the following error metrics $[23,24,25,26]$ :

$$
\begin{aligned}
& P S N R=10 \log _{10}\left(\frac{\omega_{\max }^{2}}{M S E}\right), \\
& M S E=\frac{1}{m n} \sum_{i=1}^{m} \sum_{j=1}^{n}\left(\omega_{1}^{(i j)}-\omega_{2}^{(i j)}\right)^{2},
\end{aligned}
$$

where PSNR is the Peak Signal to Noise Ratio, MSE is the Mean Square Error, $\omega_{\max }$ denotes the maximum value, e.g., for 8-bit images, $\omega_{\max }=255 ; \omega_{1}^{(i j)}$ and $\omega_{2}^{(i j)}$ are pixel values at pixel location $(i, j)$ of the image to be evaluated and of the reference image, i.e. an ideal image without any corrupted factors, respectively; $m$ and $n$ are the numbers of pixels by image width and height, respectively. Note that a difference of $0.5 \mathrm{~dB}$ is visible and higher PSNR (measured in decibels $-\mathrm{dB}$ ) indicates a better quality image.

Structural similarity (SSIM) is proven to be a suitable error metric for assessing image quality and gives a value in the range $[0,1]$, where a value closer to 1 (one) indicates better structure preservation. The SSIM between two images $\omega_{1}$ and $\omega_{2}$ of equal size $m \times n$ is computed as:

$$
\operatorname{SSIM}=\frac{\left(2 \mu_{\omega_{1}} \mu_{\omega_{2}}+c_{1}\right)\left(2 \sigma_{\omega_{1} \omega_{2}}+c_{2}\right)}{\left(\mu_{\omega_{1}}^{2}+\mu_{\omega_{2}}^{2}+c_{1}\right)\left(\sigma_{\omega_{1}}^{2}+\sigma_{\omega_{2}}^{2}+c_{2}\right)^{\prime}}
$$

where $\mu_{\omega_{i}}, \sigma_{\omega_{i}}^{2}$ are the average and the variance of $\omega_{i}, \sigma_{\omega_{1} \omega_{2}}$ is the covariance, and $c_{1}$ and $c_{2}$ are stabilization parameters.

\section{B. Dataset, Test cases and Discussion}

In the tests, we used 200 images of the UC Berkeley dataset: https://www2.eecs.berkeley.edu/Research/Projects/CS/vision/b sds/BSDS300/html/dataset/images.html, and added synthetic controlled noise to them. All images were stored in JPEG, grayscale and with various sizes. All of them are natural images.

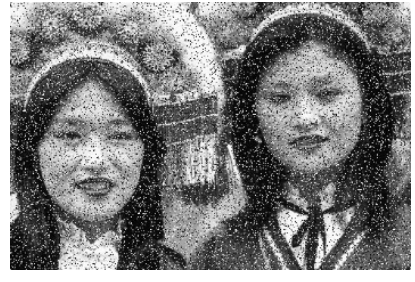

Noisy

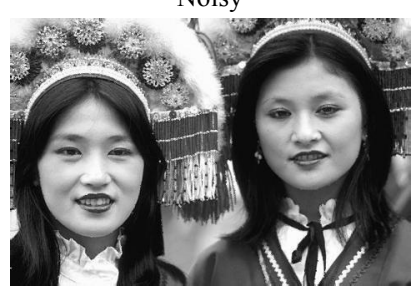

DBA

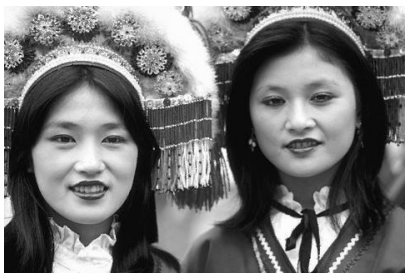

MDBUTMF

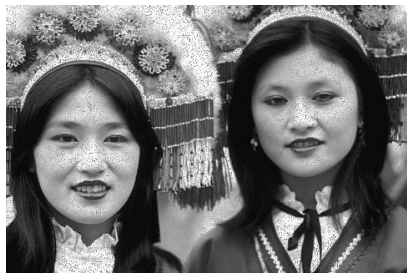

NASNLM

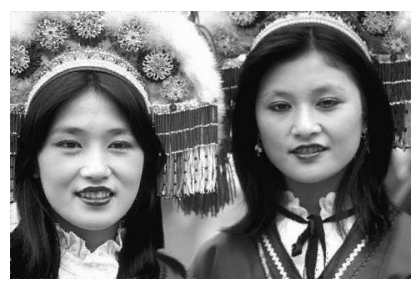

FSD

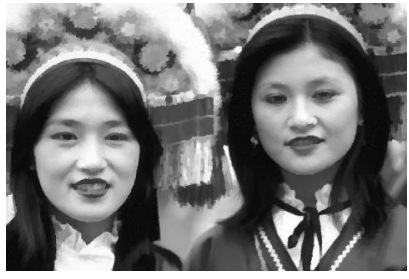

ATV1

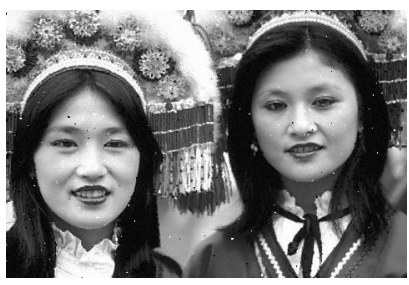

ACWMF

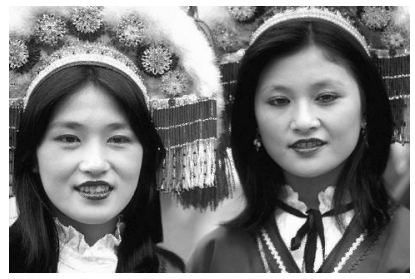

NAFSMF

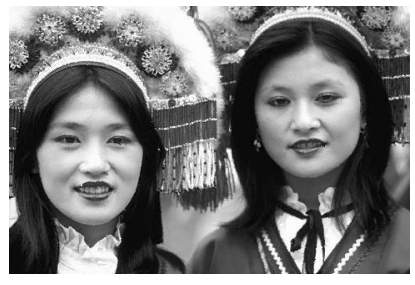

BPDF

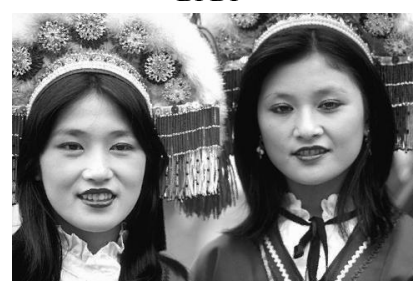

Proposed
Fig. 1. Denoising results for image " 189003 " with noise level of $20 \%$ : The PSNR/SSIM value of the noisy image is $11.6871 / 0.17778$; the denoised images by ATV1 is $24.2117 / 0.82426$, DBA is $29.7643 / 0.96243$, ACWMF is $25.2319 / 0.87549$, MDBUTMF is $30.8207 / 0.97189$, NAFSMF is $28.0891 / 0.9451$, NASNLM is $23.8167 / 0.83966$, BPDF is $28.5457 / 0.95148$, FSD is $29.7121 / 0.95921$ and the proposed method is $30.9313 / 0.97221$. 
We tested the proposed method in images with an added noise level of $10 \%, 20 \%, 30 \%, 40 \%, 50 \%, 60 \%, 70 \%, 80 \%$ and $90 \%$; and compared its denoising result against the ones obtained by state-of-the-art approaches: ATV1, DBA, ACWMF, MDBUTMF, NAFSM, NASNLM, BPDF, and FSD.

As to the first experiment, we present visual denoising results for the image " 189003 " with the noise level of $20 \%$ (Figure 1), 40\% (Figure 2), 60\% (Figure 3) and 80\% (Figure 4).

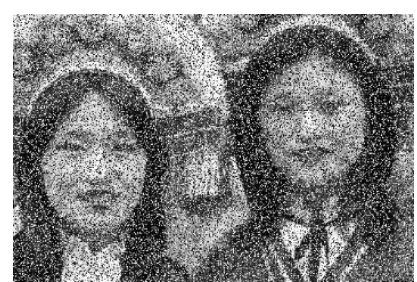

Noisy

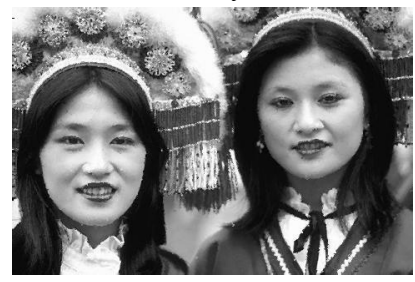

DBA

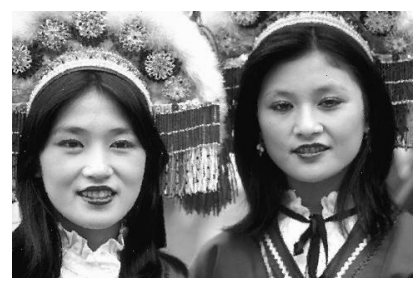

MDBUTMF

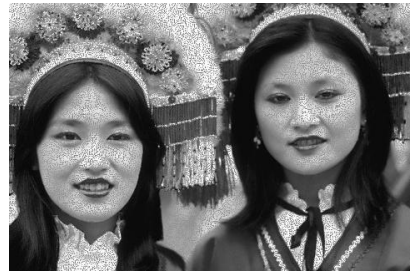

NASNLM

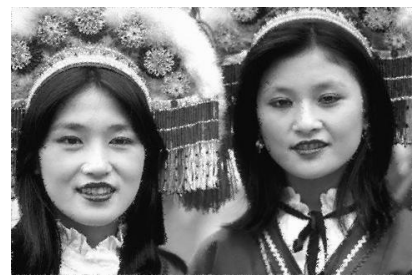

FSD

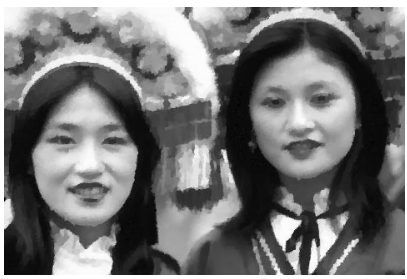

ATV1

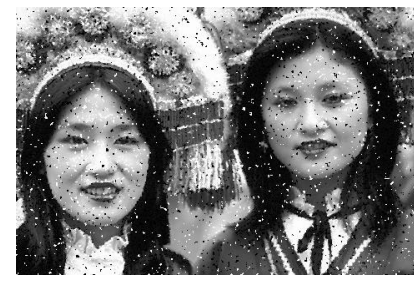

ACWMF

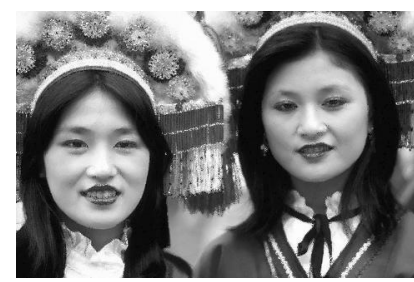

NAFSMF

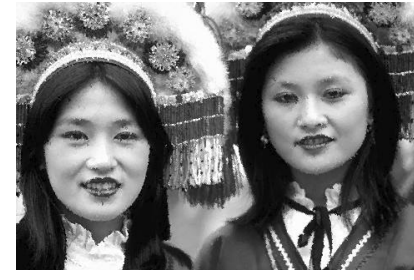

BPDF

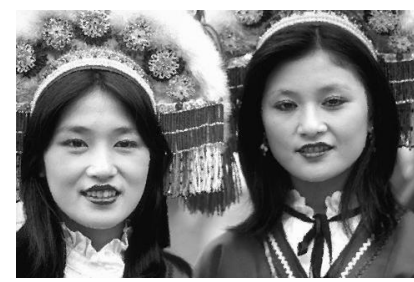

Proposed

Fig. 2. Denoising results for image " 189003 " with noise level of $40 \%$. The PSNR/SSIM value of the noisy image is $8.5836 / 0.084868$; the denoised images by ATV1 is $22.7285 / 0.77244$, DBA is $25.6268 / 0.90624$, ACWMF is $17.2833 / 0.52407$, MDBUTMF is $27.3143 / 0.93063$, NAFSMF is $25.167 / 0.89268$, NASNLM is $20.7238 / 0.75841$, BPDF is $24.2967 / 0.87242$, FSD is $25.4715 / 0.89481$ and the proposed method is $27.3823 / 0.93455$.

The obtained denoising results for the noise level of $20 \%$ are presented in Figure 1. By visual analysis, regarding the denoising result by ATV1, one can perceive that small details such as the flowers, hairs, and teeth of the women, were slightly blurred. For ACWMF and NASNLM, noise remains on the faces of the women. The denoising results by DBA, NAFSMF,

MDBUTMF, BPDF, FSD and the proposed method are better than the ones by the other methods, and it is too hard to differentiate them. The PSNR/SSIM values of the noisy image and the denoised images by ATV1, DBA, ACWMF, MDBUTMF, NAFSMF, NASNLM, BPDF, FSD and the proposed method are: $11.6871 / 0.17778,24.2117 / 0.82426$, $29.7643 / 0.96243, \quad 25.2319 / 0.87549, \quad 30.8207 / 0.97189$, 28.0891/0.9451, 23.8167/0.83966, 28.5457/0.95148, 29.7121/ $0.95921,30.9313 / 0.97221$, respectively. As can be realized, the PSNR and SSIM values confirm that our method obtained the best denoising result for this case.
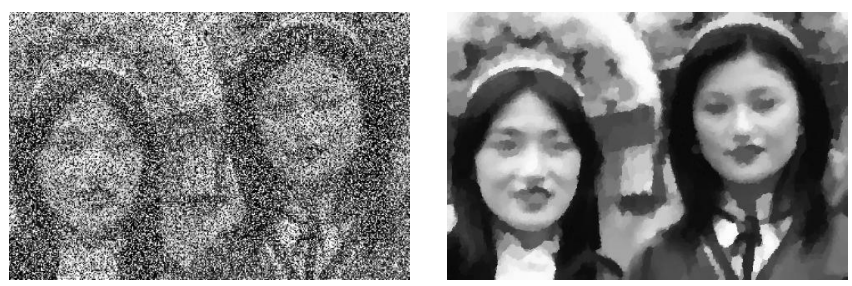
Noisy

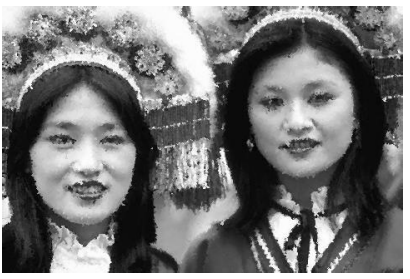

ATV 1

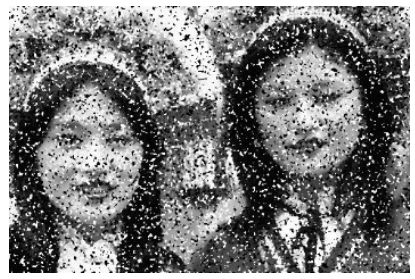

DBA

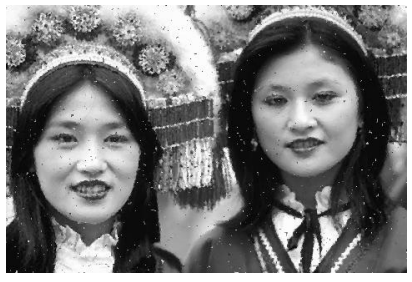
MDBUTMF

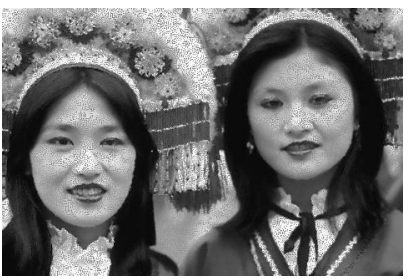
NASNLM

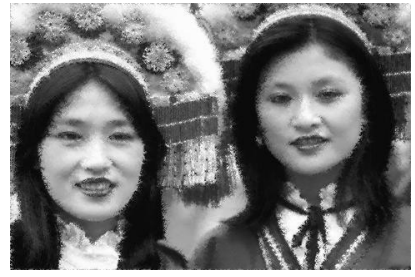

FSD

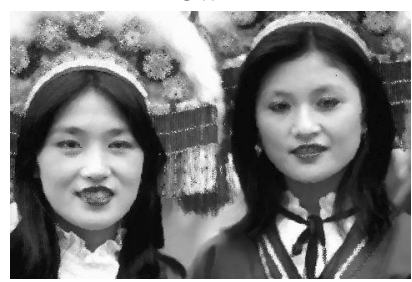

NAFSMF

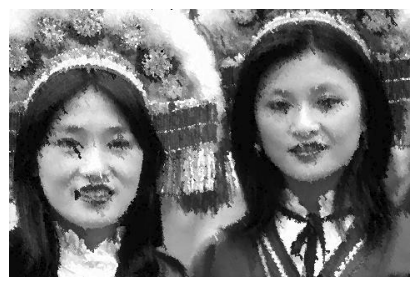

BPDF

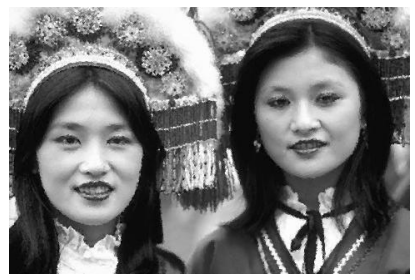

Proposed

Fig. 3. Denoising results for image " 189003 " with noise level of $60 \%$. The PSNR/SSIM value of the noisy image is 6.80623/0.042009; the denoised images by ATV1 is $20.1767 / 0.66905$, DBA is $21.6957 / 0.7956$, ACWMF is $11.1528 / 0.18071$, MDBUTMF is $23.3008 / 0.79067$, NAFSMF is $23.1409 / 0.82556$, NASNLM is $20.6894 / 0.72704$, BPDF is $20.41 / 0.72673$, FSD is $21.4187 / 0.75896$ and the proposed method is $24.6795 / 0.87751$.

As to the noise level of $40 \%$, the obtained denoising results are presented in Figure 2. In this case, many details were blurred in the denoising result by ATV1. The denoised image by NAFSMF is slightly blurred. For the cases of ACWMF and 
NASNLM, noise remains: noise on denoising result of ACWMF is more than the one of NASNLM. The denoising result by DBA contains a little noise, especially on the faces of the women. MDBUTMF removed the noise effectively. NAFSMF, BPDF, FSD and the proposed method obtained better denoising results and it is very hard to differentiate their results. The PSNR/SSIM values of the noisy image and the denoised images by ATV1, DBA, ACWMF, MDBUTMF, NAFSMF, NASNLM, BPDF, FSD and the proposed method are $8.5836 / 0.084868,22.7285 / 0.77244,25.6268 / 0.90624$, $17.2833 / 0.52407, \quad 27.3143 / 0.93063, \quad 25.167 / 0.89268$, $20.7238 / 0.75841, \quad 24.2967 / 0.87242, \quad 25.4715 / 0.89481$, $27.3823 / 0.93455$, respectively. From these values, it can be confirmed that the denoising result obtained by the proposed method is better than the ones obtained by the others.

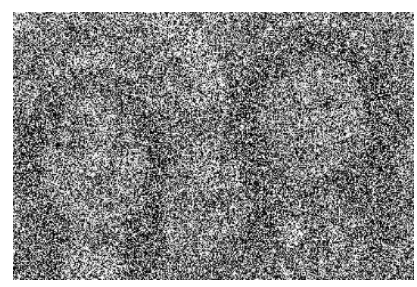

Noisy

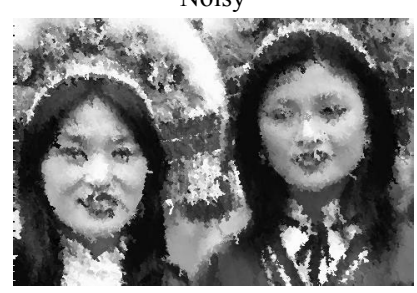

DBA

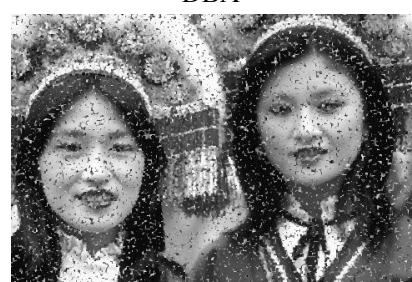

MDBUTMF

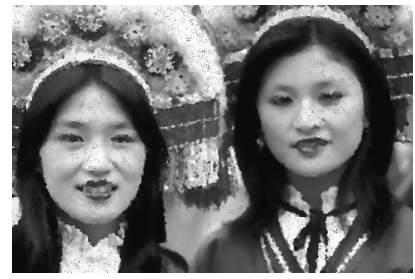

NASNLM

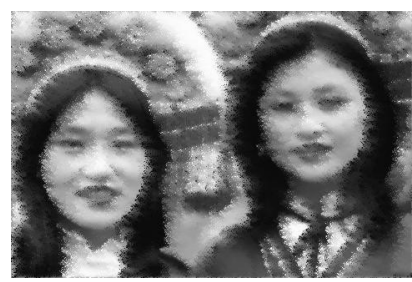

FSD

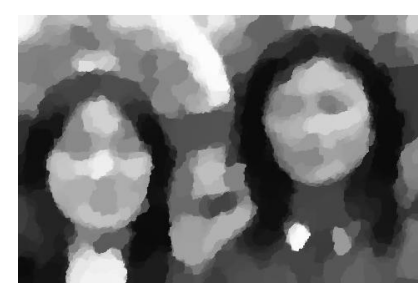

ATV1

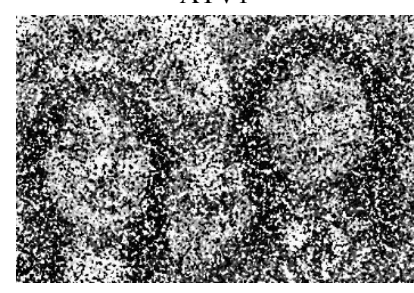

ACWMF

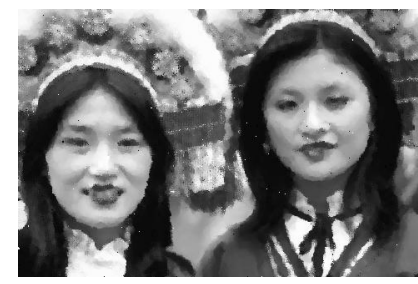

NAFSMF

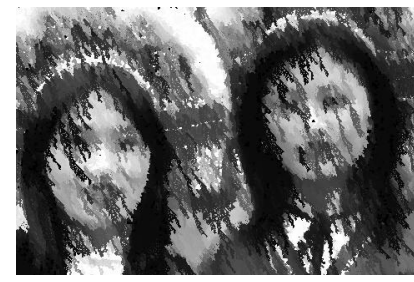

BPDF

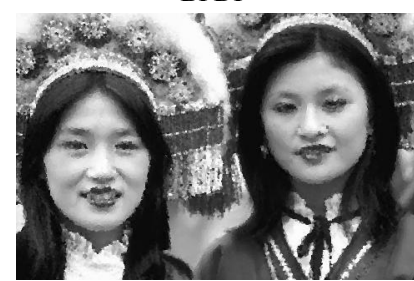

Proposed
Fig. 4. Denoising results for image " 189003 " with noise level of $80 \%$. The PSNR/SSIM value of the noisy image is 5.57764/0.018569; the denoised images by ATV1 is $16.403 / 0.50677$, DBA is $17.56 / 0.58897$, ACWMF is $7.19594 / 0.049072$, MDBUTMF is $16.5782 / 0.3789$, NAFSMF is $21.1193 / 0.72084$, NASNLM is $21.6852 / 0.72489$, BPDF is $13.8834 / 0.41728$, FSD is $16.978 / 0.50546$ and the proposed method is $21.9649 / 0.7818$.
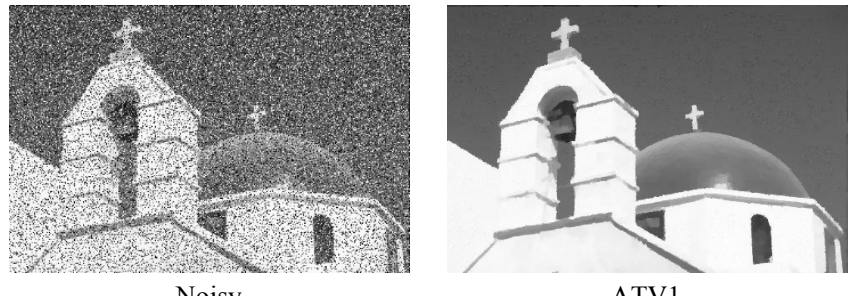

ATV1

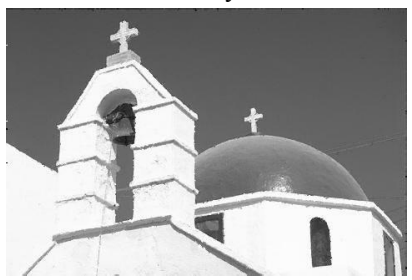

DBA

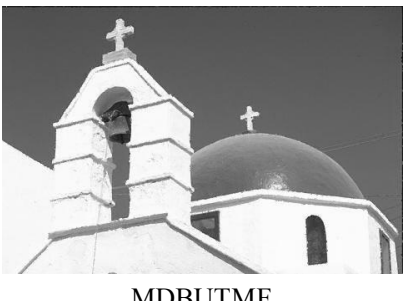

MDBUTMF

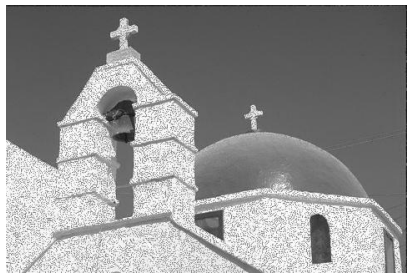

NASNLM

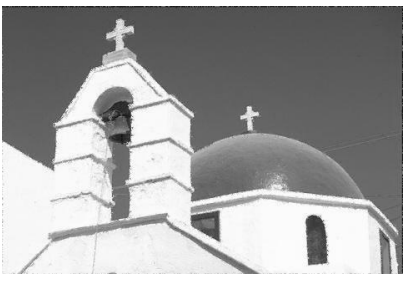

FSD

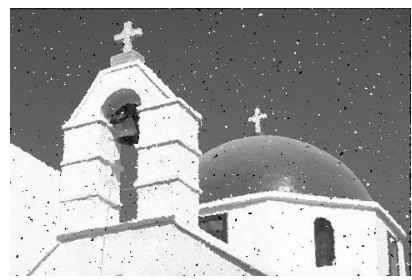

ACWMF

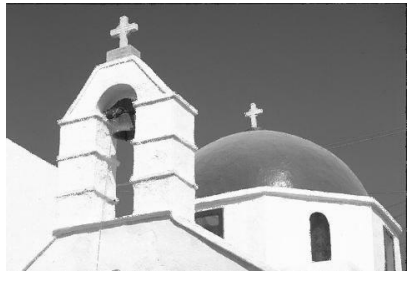

NAFSMF

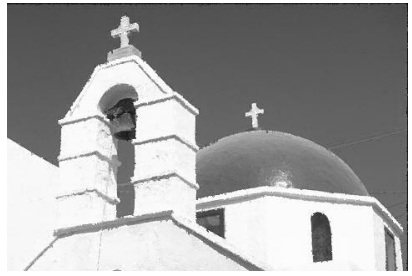

BPDF

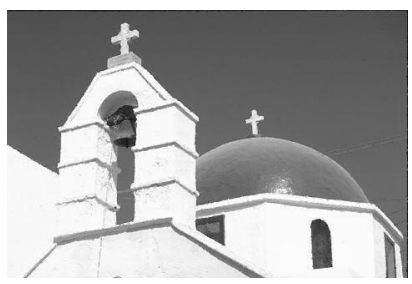

Proposed

Fig. 5. Denoising results for image " 118035 " with noise level of $30 \%$ : The PSNR/SSIM value of the noisy image is 9.9133/0.0616; the denoised images by ATV1 is $28.0023 / 0.8589$, DBA is $30.5148 / 0.9559$, ACWMF is $23.042 / 0.7565$, MDBUTMF is $33.1239 / 0.9745$, NAFSMF is $30.9115 / 0.9606$, NASNLM is $18.0177 / 0.6594$, BPDF is $29.7831 / 0.9544$, FSD is $29.9345 / 0.9486$ and the proposed method is $33.1483 / 0.9749$.

For the third case, i.e. the image with $60 \%$ of added noise, the denoising results are presented in Figure 3. The denoising result by ATV1 lost many small details: flowers, hairs, teeth, eyes. There are a lot of noises in the denoising result obtained by ACWMF. Noise remains on the denoising results by DBA, MDBUTMF, NASNLM. For NAFSMF, the denoising result is slightly blurred and the details look unnatural. BPDF caused raindrop effect. FSD did not preserve edges well. With this noise level, it is very easy to see that the denoising result by the proposed method is the best since it removed the noise effectively and preserved the details very well. The PSNR/SSIM values of the noisy image and the denoised images by ATV1, DBA, ACWMF, MDBUTMF, NAFSMF, NASNLM, BPDF, FSD and the proposed method are 
6.80623/0.042009, 11.1528/0.18071, $20.1767 / 0.66905$,

$21.6957 / 0.7956$ $24.6795 / 0.87751$, respectively. The values of PSNR and SSIM for the proposed method exceed the ones of the other methods.

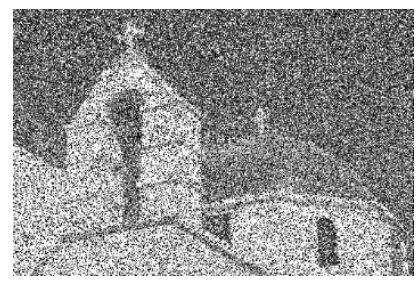

Noisy

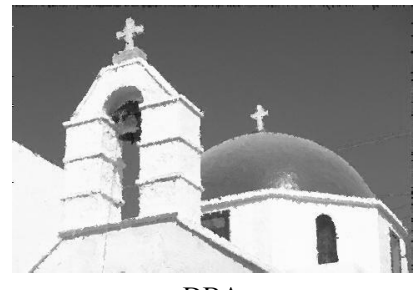

DBA

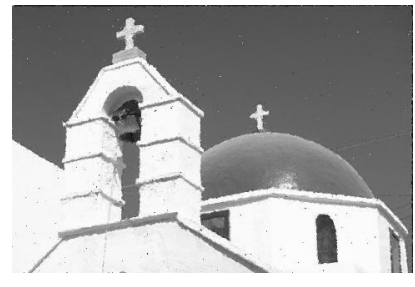

MDBUTMF

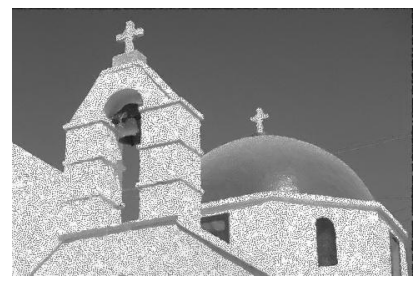

NASNLM

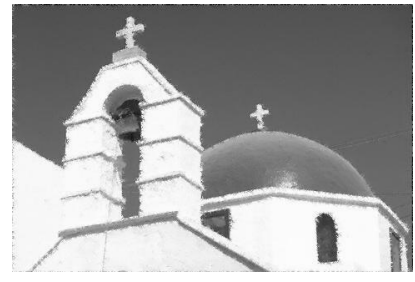

FSD

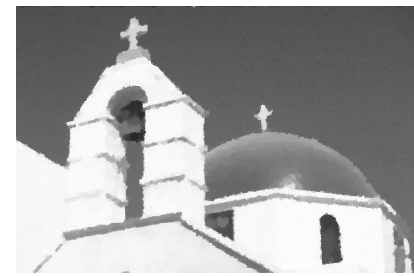

ATV1

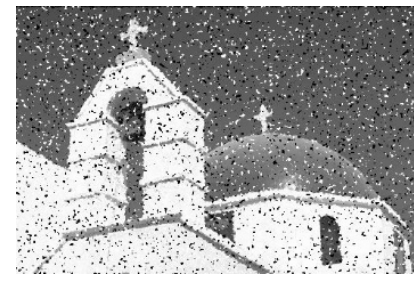

ACWMF

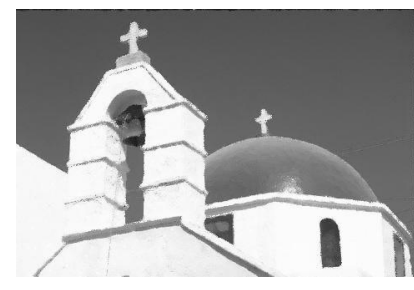

NAFSMF

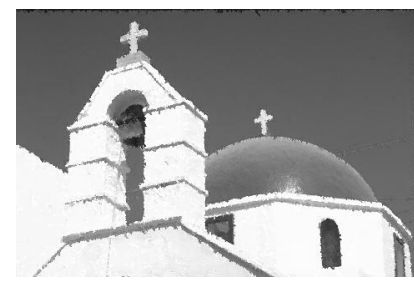

BPDF

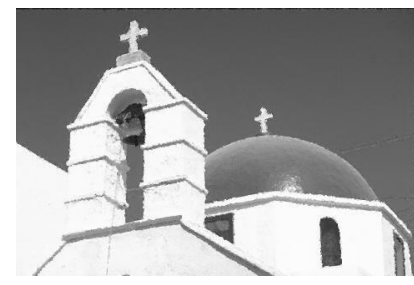

Proposed
Fig. 6. Denoising results for image " 118035 " with noise level of $50 \%$ : The PSNR/SSIM value of the noisy image is 7.6798/0.0323; the denoised images by ATV1 is $25.736 / 0.8588$, DBA is $26.9268 / 0.9165$, ACWMF is $14.4532 / 0.2436$, MDBUTMF is $28.5963 / 0.9185$, NAFSMF is $28.6065 / 0.9372$, NASNLM is $16.9479 / 0.6391$, BPDF is $25.7291 / 0.8997$, FSD is $26.3798 / 0.9017$ and the proposed method is $29.6188 / 0.9487$.

For the fourth case with $80 \%$ of added noise, the denoising results are presented in Figure 4. With this high noise level, the denoising result by ATV1 lost many details and the resulted image is too blurred. ACWMF failed. There is a lot of noise on the denoising result obtained by MDBUTMF. DBA and BPDF caused the raindrop effect. The denoising result by NASNLM contains a little noise. For NAFSMF, little noise remains and details of the denoised image are blurred. FSD blurred the contents of the image. The proposed method removed noise most effectively: removed all noises and preserved the details.
The PSNR/SSIM values of the noisy image and the denoised images by ATV1, DBA, ACWMF, MDBUTMF, NAFSMF, NASNLM, BPDF, FSD and the proposed method are 5.57764/0.018569, 16.403/0.50677, $17.56 / 0.58897$, $7.19594 / 0.049072, \quad 16.5782 / 0.3789, \quad 21.1193 / 0.72084$, 21.6852/0.72489, 13.8834/0.41728, 16.978/0.50546, $21.9649 / 0.7818$, respectively. As can be verified from these values, the values of PSNR and SSIM for the proposed method exceed the ones of the other methods.
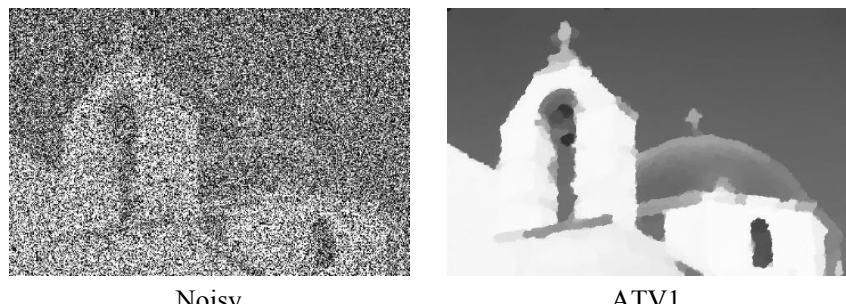
ATV1

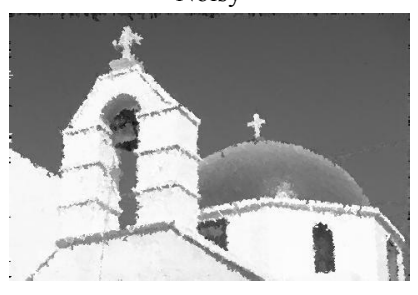

DBA

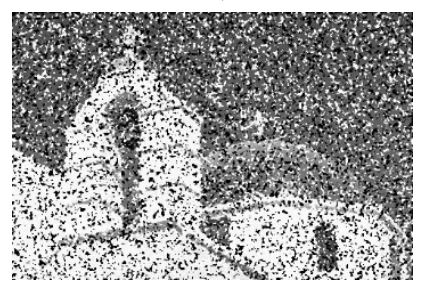

ACWMF
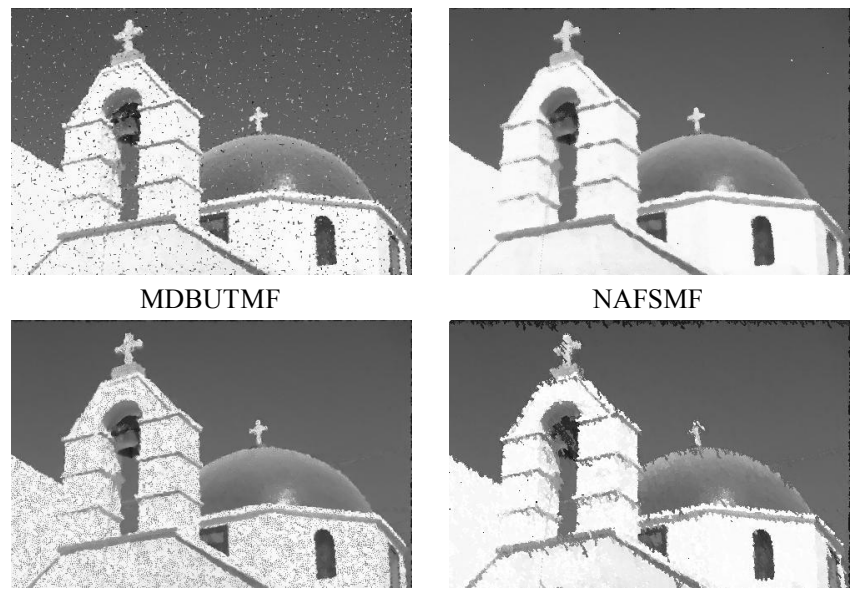

NASNLM

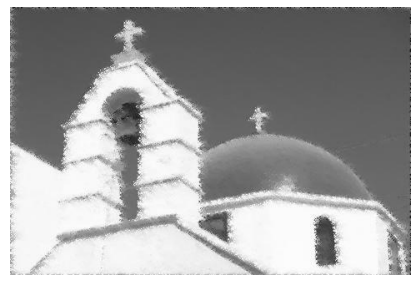

FSD

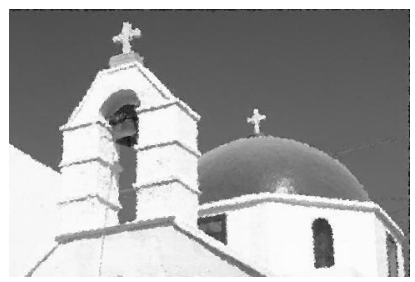

Proposed

Fig. 7. Denoising results for image " 118035 " with noise level of $70 \%$ : The PSNR/SSIM value of the noisy image is $6.2466 / 0.0162$; the denoised images by ATV1 is $21.7285 / 0.8054$, DBA is $22.9076 / 0.8428$, ACWMF is 9.3041/0.0584, MDBUTMF is $21.4104 / 0.5869$, NAFSMF is $26.0489 / 0.8975$, NASNLM is $19.3763 / 0.6502$, BPDF is $21.5104 / 0.797$, FSD is $22.3914 / 0.8157$ and the proposed method is $26.9463 / 0.9119$.

For the second experiment, we denoised image "118035" with the noise level of $30 \%$ (Figure 5 ), $50 \%$ (Figure 6), $70 \%$ (Figure 7) and $90 \%$ (Figure 8). It can be noticed that the walls of the church presented in the image are very white (near the boundary value $\delta_{\max }$ ). 
Figure 5 presents the denoising results for the image "118035" with $30 \%$ of noise. The denoising result by ATV1 is good. However, some small details such as the church bell rope is lost. There is still noise on the results of ACWMF and NASNLM. Denoising results by DBA, MDBUTMF, NAFSMF, BPDF, FSD and the proposed method are excellent. Based on the PSNR and SSIM values, one can realize that our method gave the best denoising result: PSNR/SSIM value of the noisy image is $9.9133 / 0.0616$; the denoised images by ATV1 is $28.0023 / 0.8589$, DBA is $30.5148 / 0.9559$, ACWMF is $23.042 / 0.7565$, MDBUTMF is $33.1239 / 0.9745$, NAFSMF is $30.9115 / 0.9606$, NASNLM is $18.0177 / 0.6594$, BPDF is $29.7831 / 0.9544$, FSD is $29.9345 / 0.9486$ and the proposed method is $33.1483 / 0.9749$.

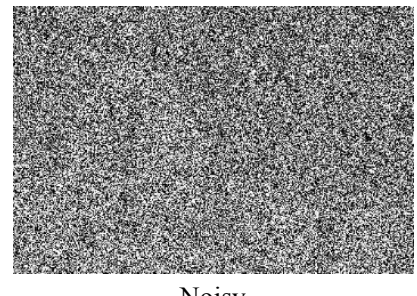

Noisy

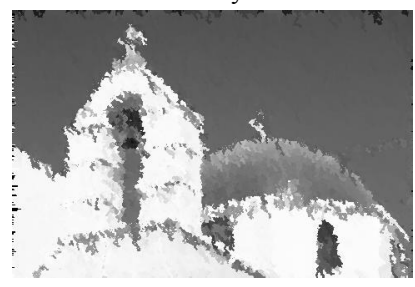

DBA

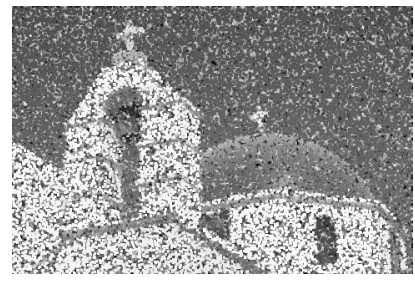

MDBUTMF

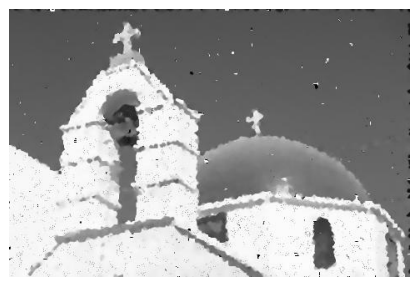

NASNLM

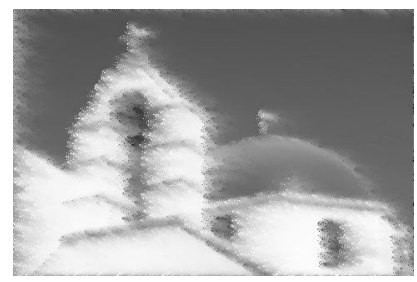

FSD

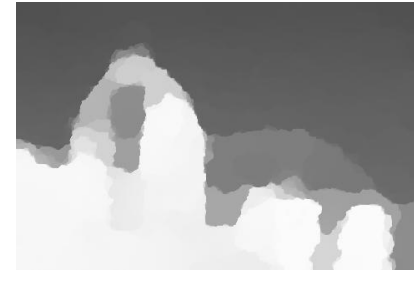

ATV1

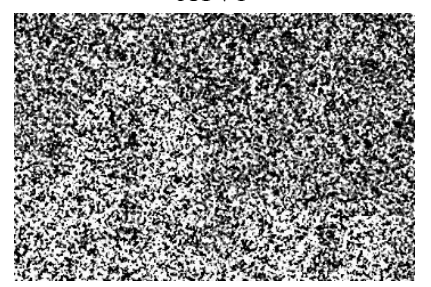

ACWMF

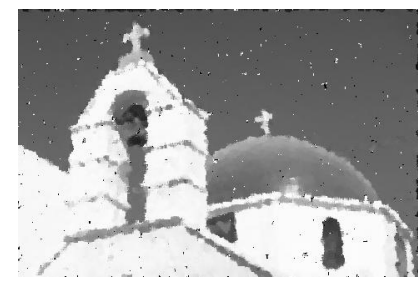

NAFSMF

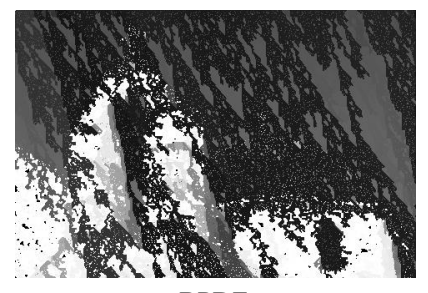

BPDF

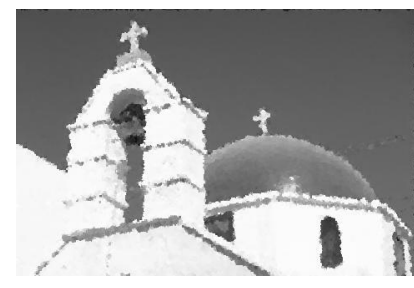

Proposed

Fig. 8. Denoising results for image " 118035 " with noise level of $90 \%$ : The PSNR/SSIM value of the noisy image is 5.1335/0.0066; the denoised images by ATV1 is $17.3135 / 0.7331$, DBA is $17.9949 / 0.7001$, ACWMF is $5.8755 / 0.013$, MDBUTMF is $13.0032 / 0.1239$, NAFSMF is $21.4361 / 0.7433$, NASNLM is $22.4325 / 0.7377$, BPDF is $8.7054 / 0.1862$, FSD is $17.1307 / 0.6328$ and the proposed method is $23.3132 / 0.8377$.

Figure 6 presents the denoising results for image "118035" with $50 \%$ of noise. Similar to the previous case, the denoising result by ATV1 lost small details: church bell-rope, small horizontal lines on the bell tower. Noise remains on the denoising result by ACWMF and NASNLM. There is a little noise in the sky of the denoising result by MDBUTMF. Edges of the church, of the bell tower, are not preserved well for the denoising results of DBA, BPDF, FSD. The denoising results by NAFSMF and the proposed method are excellent: noises were removed effectively and details and edges well preserved. The PSNR/SSIM value of the noisy image is 7.6798/0.0323; the denoised images by ATV1 is $25.736 / 0.8588$, DBA is 26.9268/0.9165, ACWMF is 14.4532/0.2436, MDBUTMF is $28.5963 / 0.9185$, NAFSMF is $28.6065 / 0.9372$, NASNLM is $16.9479 / 0.6391$, BPDF is 25.7291/0.8997, FSD is $26.3798 / 0.9017$ and the proposed method is $29.6188 / 0.9487$. As can be verified from these values, the denoising result by our method acquired the highest PSNR/SSIM value.

The denoising results for image "118035" with $70 \%$ of noise are presented in Figure 7. The denoising result by ATV1 is too smooth. There is a lot of noise in the result of ACWMF. There are a little noises in the results of MDBUTMF and NASNLM. Raindrop effect appears in the denoising result by BPDF. Edges are not preserved well for the denoising results by DBA and FSD. The denoising result by NAFSMF is good, but the details are slightly blurred and small details such as the church bell rope is lost. Otherwise, some salt pixels remain in the sky and look like artificial stars. The denoising result by the proposed method is the best, since all noises are removed, and details are preserved well. The PSNR/SSIM value of the denoising result by the proposed method is the highest: noisy image is 6.2466/0.0162; the denoised images by ATV1 is $21.7285 / 0.8054$, DBA is $22.9076 / 0.8428$, ACWMF is 9.3041/0.0584, MDBUTMF is $21.4104 / 0.5869$, NAFSMF is $26.0489 / 0.8975$, NASNLM is $19.3763 / 0.6502$, BPDF is $21.5104 / 0.797$, FSD [15] is $22.3914 / 0.8157$ and the proposed method is $26.9463 / 0.9119$.

Figure 8 presents the denoising results for image "118035" with $90 \%$ of added noise. The denoising result by ATV1 is too smooth and many details are lost. A lot of noise remains on the result of ACWMF and MDBUTMF. All contents in the denoising result by BPDF are damaged and have no meaning for restoration. Edges are not preserved well on the result by DBA. The denoising result of FSD looks that the church is in the snowstorm and many details are corrupted. NAFSMF and NASNLM removed noise effectively but caused some defects, especially artificial stars on the skin and some artefacts. Noise is removed effectively. No defect remains. The PSNR/SSIM value of the noisy image is 5.1335/0.0066; the denoised images by ATV1 is $17.3135 / 0.7331$, DBA is $17.9949 / 0.7001$, ACWMF is $5.8755 / 0.013$, MDBUTMF is $13.0032 / 0.1239$, NAFSMF is $21.4361 / 0.7433$, NASNLM is $22.4325 / 0.7377$, BPDF is $8.7054 / 0.1862$, FSD is $17.1307 / 0.6328$ and the proposed method is $23.3132 / 0.8377$. As can be confirmed by these values, the PSNR/SSIM values of the denoising result by our method are the highest, which confirms its denoising superiority. 


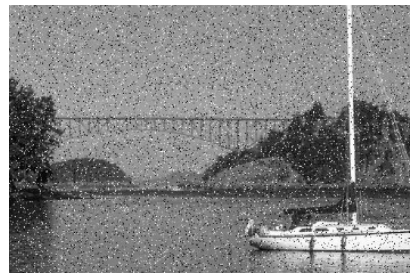

$10 \%$ of noise

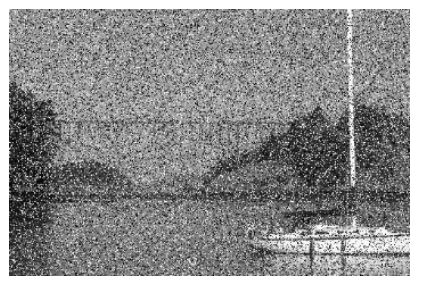

$30 \%$ of noise

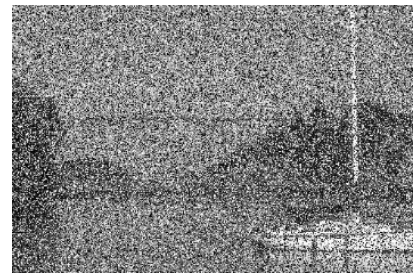

$50 \%$ of noise

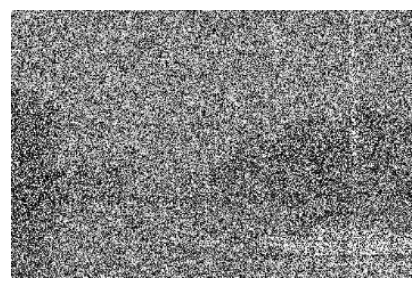

$70 \%$ of noise

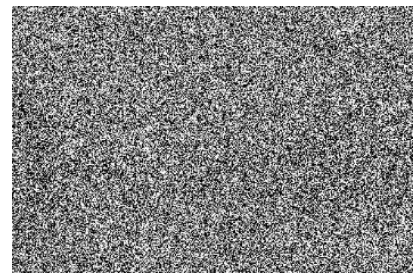

$90 \%$ of noise

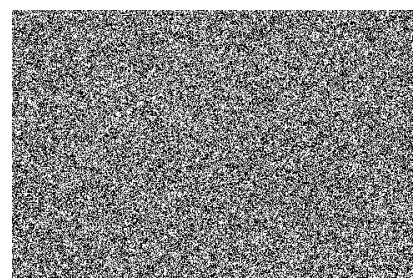

$95 \%$ of noise

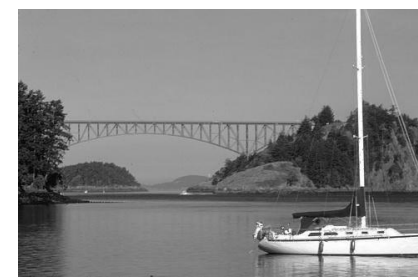

Denoised of $10 \%$ of noise

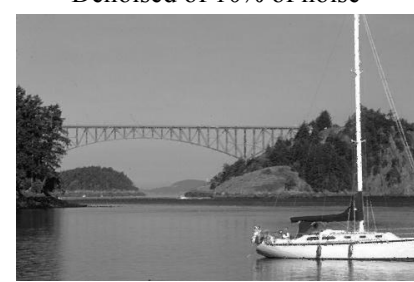

Denoised of $30 \%$ of noise

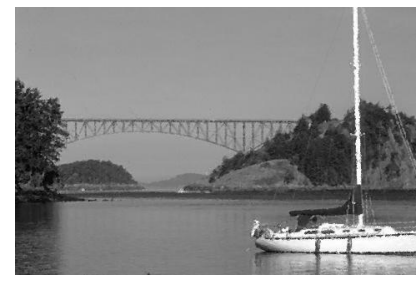

Denoised of $50 \%$ of noise

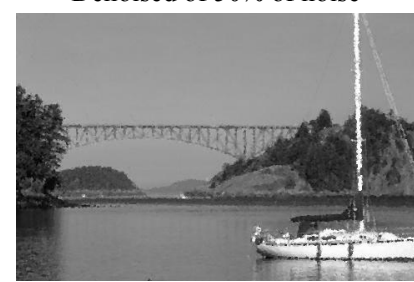

Denoised of $70 \%$ of noise

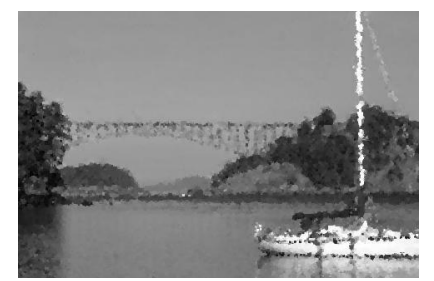

Denoised of $90 \%$ of noise

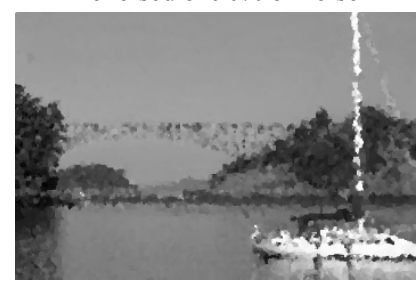

Denoised of $95 \%$ of noise
Fig. 9. Denoising results for image "22090" with various noise levels: The PSNR/SSIM value of the noisy image of $10 \%$ is $15.5246 / 0.1780$, of the denoised image of $10 \%$ is $37.5135 / 0.9900$; of the noisy image of $30 \%$ is $10.7078 / 0.0581$, of the denoised image of $30 \%$ is $32.2984 / 0.9633$; of the noisy image of $50 \%$ is $8.4886 / 0.0298$, of the denoised image of $50 \%$ is $29.0991 / 0.9273$; of the noisy image of $70 \%$ is $7.0442 / 0.0149$, of the denoised image of $70 \%$ is $26.7485 / 0.8748$; of the noisy image of $90 \%$ is $5.9508 / 0.0070$, of the denoised image of $90 \%$ is $23.3205 / 0.7748$; of the noisy image of $95 \%$ is $5.7224 / 0.0049$, of the denoised image of $95 \%$ is $21.7902 / 0.7151$.

For the second experiment, we consider the denoising results of image " 22090 " with the added noise levels of $10 \%$, $30 \%, 50 \%, 70 \%, 90 \%$, and $95 \%$. The denoising results are showed in Figure 9. With the noise levels of $10 \%$ and $30 \%$, the denoising results are perfect. It is very hard to find a defect in the resulted image. For the noise levels of $50 \%$ and $70 \%$, one can see some small defects on the mainmast. However, overall denoising result are very good. For the super-high noise levels $90 \%$ and $95 \%$, some defects appear on the mainmast and the bridge, but the main details and structures of the images are well preserved. By visual assessment, one can confirm that, the proposed method can remove effectively noise with various noise levels: from small, medium, high, very high and super high noise levels.

The average PSNR value and the average SSIM value of the denoising results for 200 natural images of the dataset are presented in Tables 1 and 2, respectively. As can be seen, with various added noise levels from 10\%, 20\%, 30\%, 40\%, 50\%, $60 \%, 70 \%, 80 \%$ and $90 \%$, the denoising results by the proposed method are better than the ones of the other methods in terms of PSNR and SSIM values. Especially, for the noise levels of $60 \%$ and $70 \%$, the PSNR and SSIM scores of the proposed method exceed far the ones obtained by the other methods.

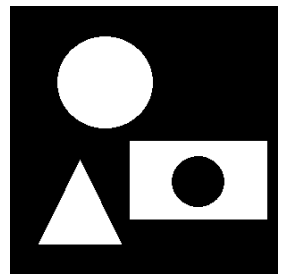

Original

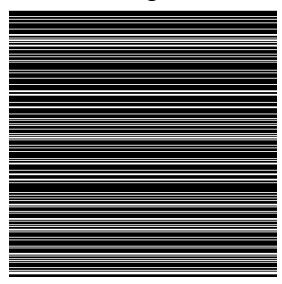

DBA

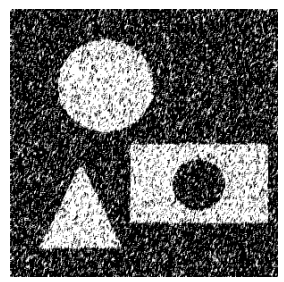

NAFSMF

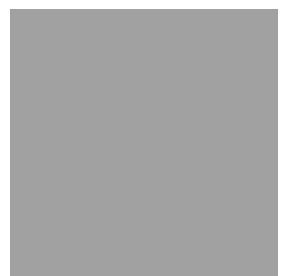

FSD

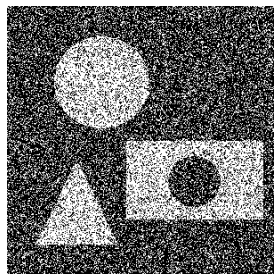

Noisy

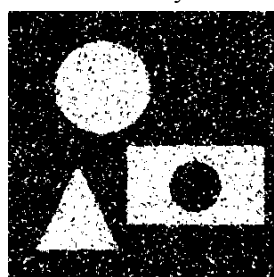

ACWMF

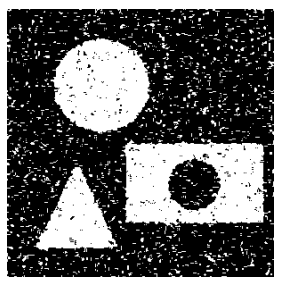

NASNLM

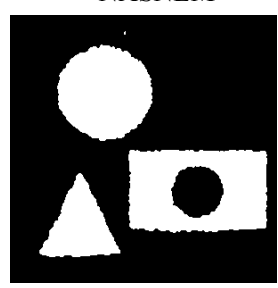

Proposed

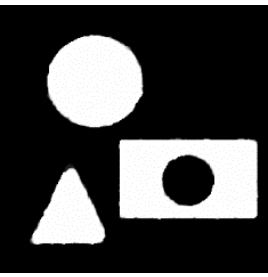

ATV1

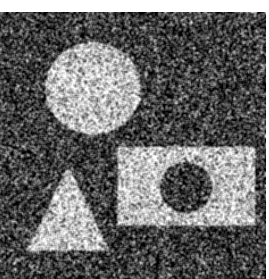

MDBUTMF

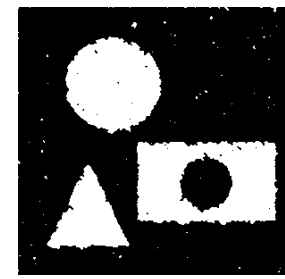

BPDF
Fig. 10. Denoising results for an artificial image with added noise level of $50 \%$ : The PSNR/SSIM value of the noisy image is 5.9804/0.0287; the denoised images by ATV1 is $23.8976 / 0.9433$, DBA is $4.3732 / 0.0877$, ACWMF is $12.4992 / 0.2827$, MDBUTMF is $10.5980 / 0.0524$, NAFSMF is $9.5650 / 0.0391$, NASNLM is $13.6689 / 0.3137$, BPDF is $17.5586 / 0.8492$, FSD is $4.8349 / 0.1849$ and the proposed method is $18.4097 / 0.9163$.

As already aforementioned, for natural images with $\mathrm{SnP}$ noise, the gray values of original pixels do not reach boundary values $\delta_{\min }, \delta_{\max }$, but only asymptote to the boundary values. 
For an 8-bit grayscale natural image, the gray value of white color is around 240 to 254 and the gray value of black color is around 1 to 15 . In this test, we built manually an artificial image of size $300 \times 300$ pixels containing only boundary values, i.e. gray value of white color is 255 and for black color is 0 (zero). It contains black background, a white circle, a white triangle, and a white rectangle bounding a black circle. We generated $\mathrm{SnP}$ noise with a noise level of $50 \%$ and applied the denoising methods under comparison.

With the built artificial image, many nonlinear filters cannot perform efficiently the denoising task. The denoising results are shown in Figure 10. Because ATV1 based on regularization that is an approach being different from other methods (nonlinear filters) and ATV1 does not use boundary values to detect noise, the denoising result by ATV1 is excellent ( $\mathrm{PSNR}=23.8976$,
SSIM $=0.9433$ ). For denoising by nonlinear filters, the denoising result by the proposed method is very good (PSNR=18.4097, SSIM=0.9163), but edges are not preserved well as the one of ATV1. However, one can see that the angles of the presented triangle are preserved better by the proposed method than by ATV1. BPDF also gives a good denoising result for this case (PSNR=17.5586, SSIM=0.8492). There are a lot of noise in the denoising results by ACWMF ( $\mathrm{PSNR}=12.4992$, $\mathrm{SSIM}=0.2827$ ), MDBUTMF (PSNR=10.5980, $\mathrm{SSIM}=0.0524$ ), NAFSMF (PSNR $=9.5650, \quad \mathrm{SSIM}=0.0391)$ and NASNLM (PSNR=13.6689, SSIM=0.3137). It could be noted that the methods DBA and FSD did not obtain a meaningful restoration. Among the nonlinear filters, the proposed method achivied the best denoising result.

TABLE 1. Average PSNR values obtained by the denoising methods for 200 natural images.

\begin{tabular}{|c|c|c|c|c|c|c|c|c|c|}
$\begin{array}{r}\text { Noise } \\
\text { Methods }\end{array}$ & $\mathbf{1 0 \%}$ & $\mathbf{2 0 \%}$ & $\mathbf{3 0 \%}$ & $\mathbf{4 0 \%}$ & $\mathbf{5 0 \%}$ & $\mathbf{6 0 \%}$ & $\mathbf{7 0 \%}$ & $\mathbf{8 0 \%}$ & $\mathbf{9 0 \%}$ \\
\hline Noisy & 15.1308 & 12.1244 & 10.3654 & 9.1148 & 8.1468 & 7.3521 & 6.6832 & 6.1032 & 5.5927 \\
\hline ATV1 & 27.2466 & 26.5406 & 25.8655 & 25.1816 & 24.4511 & 23.2259 & 21.9856 & 20.6002 & 18.7985 \\
\hline DBA & 35.7290 & 32.5830 & 30.2580 & 28.2929 & 26.5454 & 24.7821 & 22.9734 & 21.0028 & 18.4629 \\
\hline ACWMF & 32.1585 & 27.3530 & 22.4144 & 18.1337 & 14.6430 & 11.8371 & 9.5863 & 7.7616 & 6.3017 \\
\hline MDBUTMF & 37.0194 & 33.7747 & 31.6289 & 29.7897 & 27.8424 & 25.3145 & 22.0870 & 18.3913 & 14.5920 \\
\hline NAFSMF & 33.7353 & 31.0020 & 29.2642 & 27.9661 & 26.8885 & 25.9104 & 24.9053 & 23.7189 & 21.0489 \\
\hline NASNLM & 32.4355 & 28.9482 & 26.8255 & 25.5203 & 24.8363 & 24.6808 & 24.7947 & 24.6729 & 22.4444 \\
\hline BPDF & 35.3026 & 31.7015 & 29.2786 & 27.2985 & 25.4366 & 23.5613 & 21.3846 & 17.9047 & 12.1222 \\
\hline FSD & 35.5074 & 32.4981 & 30.2457 & 28.2938 & 26.4564 & 24.7208 & 23.0033 & 21.1793 & 18.8880 \\
\hline DAMF & 37.0100 & 33.8100 & 31.7200 & 30.0500 & 28.6500 & 27.3600 & 26.0800 & 24.6600 & 22.6400 \\
\hline AFMF & 32.3500 & 31.2900 & 30.1200 & 28.8900 & 27.6500 & 26.4100 & 25.1700 & 23.8200 & 19.7100 \\
\hline Proposed & $\mathbf{3 7 . 1 2 6 0}$ & $\mathbf{3 3 . 9 6 9 4}$ & $\mathbf{3 1 . 8 2 0 1}$ & $\mathbf{3 0 . 1 5 6 1}$ & $\mathbf{2 8 . 7 5 2 3}$ & $\mathbf{2 7 . 4 0 9 1}$ & $\mathbf{2 6 . 1 4 0 2}$ & $\mathbf{2 4 . 7 6 5 5}$ & $\mathbf{2 2 . 9 2 1 9}$ \\
\hline
\end{tabular}

TABLE 2. Average SSIM values obtained by the denoising methods for 200 natural images.

\begin{tabular}{|c|c|c|c|c|c|c|c|c|c|}
\hline $\begin{array}{c}\text { Noise } \\
\text { Mevel }\end{array}$ & $\mathbf{1 0 \%}$ & $\mathbf{2 0 \%}$ & $\mathbf{3 0 \%}$ & $\mathbf{4 0 \%}$ & $\mathbf{5 0 \%}$ & $\mathbf{6 0 \%}$ & $\mathbf{7 0 \%}$ & $\mathbf{8 0 \%}$ & $\mathbf{9 0 \%}$ \\
\hline Noisy & 0.2452 & 0.1313 & 0.0852 & 0.0593 & 0.0423 & 0.0301 & 0.021 & 0.0138 & 0.0080 \\
\hline ATV1 & 0.7807 & 0.7548 & 0.7303 & 0.7043 & 0.6750 & 0.6151 & 0.5646 & 0.5199 & 0.4766 \\
\hline DBA & 0.9789 & 0.9576 & 0.9300 & 0.8944 & 0.8489 & 0.7899 & 0.7143 & 0.6164 & 0.4859 \\
\hline ACWMF & 0.9555 & 0.8852 & 0.7220 & 0.4839 & 0.2683 & 0.1361 & 0.0683 & 0.0335 & 0.0148 \\
\hline MDBUTMF & 0.9830 & 0.9653 & 0.9443 & 0.9170 & 0.8728 & 0.7807 & 0.6052 & 0.3682 & 0.1649 \\
\hline NAFSMF & 0.9695 & 0.9408 & 0.9108 & 0.8786 & 0.8432 & 0.8029 & 0.7548 & 0.6918 & 0.5661 \\
\hline NASNLM & 0.9382 & 0.8850 & 0.8402 & 0.8034 & 0.7748 & 0.7540 & 0.7385 & 0.7200 & 0.6503 \\
\hline BPDF & 0.9774 & 0.9503 & 0.9164 & 0.8727 & 0.8174 & 0.7453 & 0.6491 & 0.5038 & 0.2974 \\
\hline FSD & 0.9778 & 0.9557 & 0.9280 & 0.8913 & 0.8425 & 0.7795 & 0.7010 & 0.6052 & 0.4892 \\
\hline DAMF & 0.9837 & 0.9663 & 0.9460 & 0.9217 & 0.8931 & 0.8588 & 0.8155 & 0.7566 & 0.6582 \\
\hline AFMF & 0.9444 & 0.9367 & 0.9227 & 0.9019 & 0.8732 & 0.8352 & 0.7854 & 0.7174 & 0.5760 \\
\hline Proposed & $\mathbf{0 . 9 8 4 3}$ & $\mathbf{0 . 9 6 7 1}$ & $\mathbf{0 . 9 4 6 5}$ & $\mathbf{0 . 9 2 2 3}$ & $\mathbf{0 . 8 9 3 5}$ & $\mathbf{0 . 8 5 8 8}$ & $\mathbf{0 . 8 1 6 0}$ & $\mathbf{0 . 7 5 8 6}$ & $\mathbf{0 . 6 6 4 8}$ \\
\hline
\end{tabular}

TABLE 3. Average execution time (second) obtained by the denoising methods for 200 natural images.

\begin{tabular}{|c|c|c|c|c|c|c|c|c|c|}
\hline $\begin{array}{r}\text { Noise } \\
\text { Methods }\end{array}$ & $\mathbf{1 0 \%}$ & $\mathbf{2 0 \%}$ & $\mathbf{3 0 \%}$ & $\mathbf{4 0 \%}$ & $\mathbf{5 0 \%}$ & $\mathbf{6 0 \%}$ & $\mathbf{7 0 \%}$ & $\mathbf{8 0 \%}$ & $\mathbf{9 0 \%}$ \\
\hline ATV1 & 11.7223 & 11.0564 & 10.9267 & 12.3502 & 11.7211 & 11.126 & 11.1933 & 10.7911 & 9.9408 \\
\hline DBA & 4.1100 & 3.8795 & 3.9902 & 4.8314 & 4.1245 & 4.0539 & 4.2624 & 3.9391 & 3.3840 \\
\hline ACWMF & 0.3125 & 0.3065 & 0.2991 & 0.3326 & 0.3446 & 0.3234 & 0.3116 & 0.3045 & 0.2656 \\
\hline MDBUTMF & 0.6513 & 1.1581 & 1.5861 & 2.5265 & 2.8469 & 2.9981 & 3.5268 & 3.5673 & 2.8597 \\
\hline NAFSMF & 1.3825 & 2.5575 & 3.7608 & 5.5110 & 6.7897 & 7.3962 & 8.5141 & 9.5143 & 9.7087 \\
\hline NASNLM & 2.3902 & 4.6452 & 6.0279 & 10.0299 & 12.974 & 14.3777 & 16.1169 & 18.1122 & 17.5409 \\
\hline BPDF & 0.8616 & 1.6022 & 2.2575 & 3.5935 & 4.5931 & 4.6425 & 5.4828 & 6.1797 & 5.7972 \\
\hline FSD & 0.9079 & 1.7548 & 2.6247 & 4.6846 & 6.7567 & 7.9926 & 10.4534 & 12.7221 & 13.3799 \\
\hline DAMF & 0.1900 & 0.3200 & 0.4900 & 0.6600 & 0.7800 & 0.9600 & 1.1300 & 1.3100 & 1.5800 \\
\hline AFMF & 6.4300 & 6.8700 & 6.5800 & 6.3500 & 7.8200 & 7.4900 & 7.1800 & 7.5000 & 8.0600 \\
\hline Proposed & 0.1952 & 0.3477 & 0.5096 & 0.7170 & 0.9307 & 1.0056 & 1.1483 & 1.3950 & 1.4849 \\
\hline
\end{tabular}


Table 3 presents the execution time of the methods under comparison for various noise levels. Figure 11 shows the change of execution time in terms of the noise levels. As one can verify, the execution times of ATV1 and ACWMF just vary a little for various noise levels. The execution times of NASNLM, FSD, NAFSMF are highly dependent on the noise levels. Overall, ACWMF is the fastest and followed by DAMF and the proposed method. The difference in the execution time of DAMF and the proposed method is slight. Moreover, for the noise level of $10 \%$, the proposed method is still faster than ACWMF. One could noticed that for noise levels of $50 \%$ or higher, ACWMF works ineffectively.

Figure 12 presents the average execution times of the methods under comparison for all considered noise levels. As the results indicated, the ACWMF and the proposed method work much faster than the others. The NASNLM is the slowest. To process noise on color images, we can easily extend the proposed method by implementing the denoising on separate image color channels. In addition to the proposed method be simple, it is easy to be parallelly processed, which is very attractive to improve its computational processing speed.

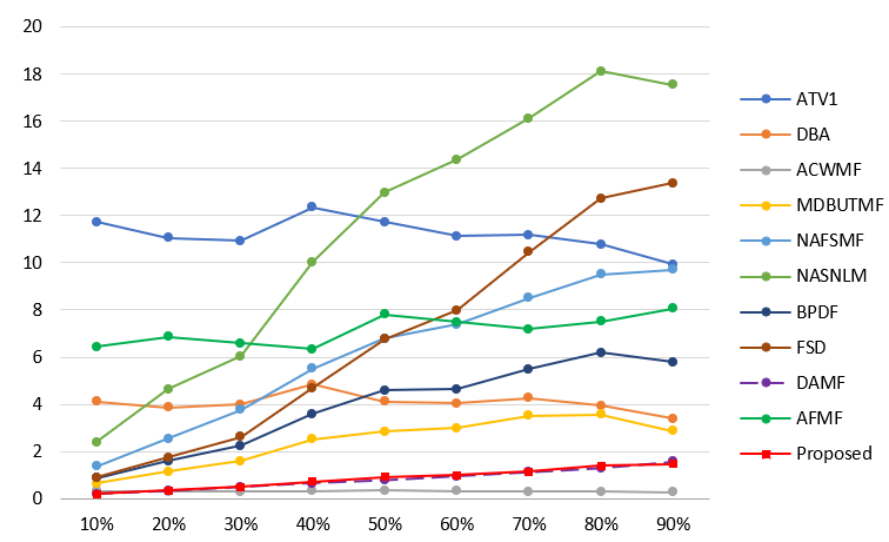

Fig. 11. Change of execution time in terms of the noise level

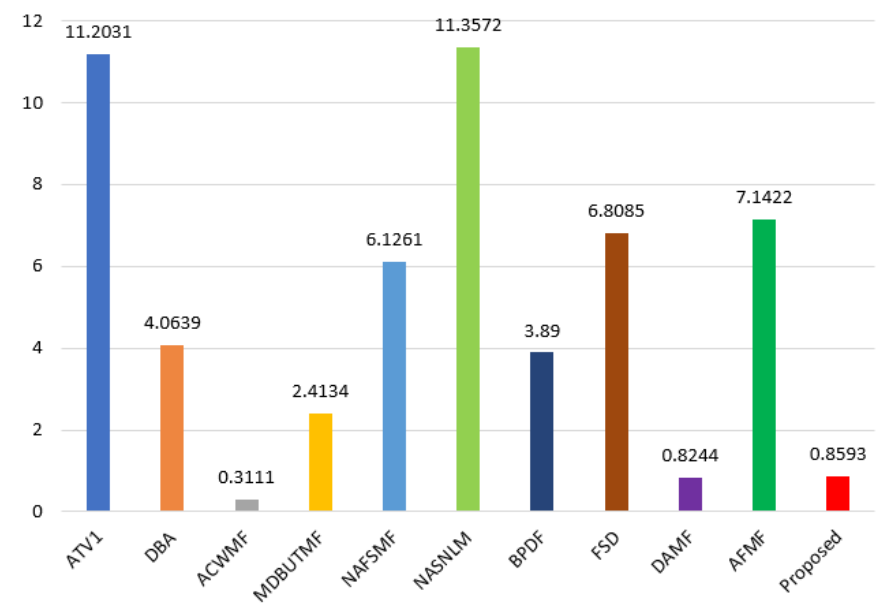

Fig. 12. Average execution time (second) of the methods for the noise levels $(10 \%, 20 \%, 30 \%, 40 \%, 50 \%, 60 \%, 70 \%, 80 \%, 90 \%)$ for 200 natural images.

\section{CONCLUSIONS}

In this article, we proposed a two-stage filter based to remove high-density salt and pepper noise in images. The proposed method works effectively for various noise levels: low-density, medium-density, high-density, and super-high-density noises. As could be confirmed by the PSNR and SSIM metrics, the achieved denoising results are remarkable. The proposed method can work excellent for denoising $\mathrm{SnP}$ noise on natural images, as well as for artificial images with gray value of pixels being boundary values. Otherwise, it also preserves details and other image structures outstandingly. The proposed method is non-iterative, and can perform very fast, which is a very attractive advantage to process large images or video sequences. In future, we plan to extend the proposed method to denoise also random-value impulse noise and other noises in images. Aditionally, the aplication of the proposed method combined with deep learning models, in order to solve the problem of detecting salient objects [27] in images degraded by noise, is an interesting problem to be explored.

\section{REFERENCES}

[1] R. Rojas and P. Rodriguez, "Spatially Adaptive Total Variation Image Denoising Under Salt and Pepper noise," in 19th European Signal Processing Conference EUSIPCO, pp 278-282, Barcelona, 2011.

[2] D. N. H. Thanh, V. B. S. Prasath and L. T. Thanh, "Total Variation L1 Fidelity Salt-and-Pepper Denoising with Adaptive Regularization Parameter," in IEEE The 5th NAFOSTED Conference on Information and Computer Science (NICS), Ho Chi Minh, 2018.

[3] T. F. Chan and S. Esedoglu, "Aspects of Total Variation Regularized L1 Function Approximation," SIAM Journal on Applied Mathematics, vol. 65, no. 5, p. 1817-1837, 2005.

[4] T. Goldstein and S. Osher, "The split Bregman method for L1 regularized problem," SIAM Journal on Imaging Sciences, vol. 2, no. 2, pp. 323-343, 2009.

[5] D. N. H. Thanh, L. T. Thanh, N. N. Hien and S. Prasath, "Adaptive total variation L1 regularization for salt and pepper image denoising," Optik, 2020 (In press). Doi: 10.1016/j.ijleo.2019.163677.

[6] R. H. Chan, C.-W. Ho and M. Nikolova, "Salt-and-pepper noise removal by median-type noise detectors and detailpreserving regularization," IEEE Transactions on Image Processing, vol. 14, no. 10, pp. 1479-1485, 2005.

[7] J.-H. Lin, N. Ansari and J. Li, "Nonlinear filtering by threshold decomposition," IEEE Transactions on Image Processing, vol. 8, no. 7, pp. 925 - 933, 1999.

[8] C.-H. Lin, J.-S. Tsai and C.-T. Chiu, "Switching Bilateral Filter With a Texture/Noise Detector for Universal Noise Removal," IEEE Transactions on Image Processing, vol. 19, no. 9, pp. 2307 - 2320, 2010.

[9] S. Enginoglu, U. Erkan and S. Memis, "Pixel similaritybased adaptive Riesz mean filter for salt-and-pepper 
noise removal," Multimedia Tools and Applications, vol. 78, p. 35401-35418, 2019.

[10] H. Hwang and R. A. Haddad, "Adaptive median filters: New algorithms and results," IEEE Transaction on Image Processing, vol. 4, no. 4, p. 499-502, 1995.

[11] K. K. V. Toh and N. A. M. Isa, "Noise Adaptive Fuzzy Switching Median Filter for Salt-and-Pepper Noise Reduction," IEEE Signal Processing Letters, vol. 17, no. 3, pp. 281-284, 2010.

[12] K. Aiswarya, V. Jayaraj and D. Ebenezer, "A New and Efficient Algorithm for the Removal of High Density Salt and Pepper Noise in Images and Videos," in IEEE 2nd International Conference on Computer Modeling and Simulation, Sanya, 2010.

[13] T. Bai and J. Tan, "Automatic detection and removal of high-density impulse noises," IET Image Processing, vol. 9, no. 2, pp. 162-172, 2015.

[14] S. Esakkirajan, T. Veerakumar, A. N. Subramanyam and C. H. PremChand, "Removal of High Density Salt and Pepper Noise Through Modified Decision Based Unsymmetric Trimmed Median Filter," IEEE Signal Processing Letters, vol. 18, no. 5, pp. 287-290, 2011.

[15] U. Erkan and L. Gokrem, "A new method based on pixel density in salt and pepper noise removal," Turkish Journal of Electrical Engineering \& Computer Sciences, vol. 26, pp. 162-171, 2018.

[16] U. Erkan, L. Gokrem and S. Enginoglu, "Different applied median filter in salt and pepper noise," Computers and Electrical Engineering, vol. 70, pp. 789798, 2018.

[17] U. Erkan, S. Enginoglu, D. N. H. Thanh and L. M. Hieu, "Adaptive Frequency Median Filter for the Salt-andPepper Denoising Problem," IET Image Processing, 2020 (In press). Doi: 10.1049/iet-ipr.2019.0398

[18] U. Erkan, D. N. H. Thanh, L. M. Hieu and S. Enginoglu, "An Iterative Mean Filter for Image Denoising," IEEE Access, vol. 7, no. 1, pp. 167847 - 167859, 2020.

[19] K. S. Srinivasan and D. Ebenezer, "A New Fast and Efficient Decision-Based Algorithm for Removal of
High-Density Impulse Noises," IEEE Signal Processing Letters, vol. 14, no. 3, pp. 189 - 192, 2007.

[20] T. Chen and H. R. Wu, "Adaptive impulse detection using center-weighted median filters," IEEE Signal Processing Letters, vol. 8, no. 1, pp. 1-3, 2001.

[21] J. Varghese, N. Tairan and S. Subash, "Adaptive Switching Non-local Filter for the Restoration of Salt and Pepper Impulse-Corrupted Digital Images," Arabian Journal for Science and Engineering, vol. 40, no. 11, p. 3233-3246, 2015.

[22] V. Singh, R. Dev, N. K. Dhar, P. Agrawal and N. K. Verma, "Adaptive type-2 fuzzy approach for filtering salt and pepper noise in grayscale images," IEEE Transactions on Fuzzy Systems , vol. 26, no. 5, pp. 3170 - 3176, 2018.

[23] Z. Wang, A. Bovik, H. Sheikh, Simoncelli and Eero, "Image quality assessment: From error visibility to structural similarity," IEEE Transactions on Image Processing, vol. 13, no. 4, pp. 600-612, 2004.

[24] D. N. H. Thanh and S. Dvoenko, "A method of total variation to remove the mixed Poisson-Gaussian noise," Pattern Recognition and Image Analysis, vol. 26, no. 2, pp. 285-293, 2016.

[25] V. B. S. Prasath, D. Vorotnikov, R. Pelapur, S. Jose, Seetharaman, G. and K. Palaniappan, "Multiscale Tikhonov-Total Variation Image Restoration Using Spatially Varying Edge Coherence Exponent," IEEE Transactions on Image Processing, vol. 24, no. 12, pp. 5220-5235, 2015.

[26] V. B. S. Prasath, R. Pelapur, G. Seetharaman and K. Palaniappan, "Multiscale structure tensor for improved feature extraction and image regularization," IEEE Transactions on Image Processing, 2019.

[27] J. X. Zhao, J. J. Liu, D. P. Fan, Y. Cao, J. Yang and M. M. Chen, "EGNet: Edge Guidance Network for Salient Object Detection," in International Conference on Computer Vision (ICCV), Seoul, 2019. 